\title{
Téoros
}

Revue de recherche en tourisme

\section{De la mise en valeur du patrimoine au développement durable}

\section{Martine Freedman, Thomas Antil et Jean-Pierre Gagnon}

Volume 30, numéro 2, 2011

URI : https://id.erudit.org/iderudit/1012249ar

DOI : https://doi.org/10.7202/1012249ar

Aller au sommaire du numéro

Éditeur(s)

Université du Québec à Montréal

ISSN

0712-8657 (imprimé)

1923-2705 (numérique)

Découvrir la revue

Citer cet article

Freedman, M., Antil, T. \& Gagnon, J.-P. (2011). De la mise en valeur du patrimoine au développement durable. Téoros, 30(2), 124-127.

https://doi.org/10.7202/1012249ar d'utilisation que vous pouvez consulter en ligne. 


\section{De la mise en valeur du patrimoine au développement durable ${ }^{1}$}

\author{
Martine FREEDMAN, Ph.D. \\ Direction de l'évaluation et de l'analyse stratégique \\ Ministère du Tourisme \\ martine.freedman@tourisme.gouv.qc.ca
}

Thomas ANTIL, M.A.

Direction de l'évaluation et de l'analyse stratégique

Ministère du Tourisme

thomas.antil@tourisme.gouv.qc.ca

\author{
Jean-Pierre GAGNON, Ph.D. \\ Direction de l'évaluation et de l'analyse stratégique \\ Ministère du Tourisme \\ jean-pierre.gagnon@tourisme.gouv.qc.ca
}

Dans cette première chronique, nous aborderons, d'une part, l'importance du patrimoine dans les pratiques des touristes et, d'autre part, la nécessité de considérer le développement durable dans les pratiques touristiques liées au patrimoine. Le ministère de la Culture du Québec (2011) a défini le patrimoine culturel comme les personnages, les lieux et les événements historiques, les documents, les immeubles, les objets et les sites patrimoniaux, les paysages culturels patrimoniaux et le patrimoine immatériel. Notons que les éléments naturels peuvent être pris en compte lorsque le patrimoine est considéré comme "tout objet ou ensemble, naturel ou culturel, matériel ou immatériel, qu'une collectivité reconnaît pour ses valeurs de témoignage et de mémoire historique en faisant ressortir la nécessité de le protéger, de le conserver, de se l'approprier, de le mettre en valeur et de le transmettre» (Ville de Montréal, 2004 : 4).

Plusieurs études montrent que le tourisme culturel, dans lequel s'inscrivent les activités liées au patrimoine, constitue une part non négligeable des buts des voyageurs. Tel est le cas d'une étude menée par l'OCDE qui montre que le voyage culturel représente $40 \%$ de l'ensemble du tourisme international en 2007, tendance qui devrait d'ailleurs se maintenir, voire augmenter en raison du vieillissement de la population et de l'intérêt croissant pour la culture en tant que source d'identité et de différentiation en réaction à la mondialisation (OCDE, 2009).

Par ailleurs, relevons l'apport de ce type de tourisme sur le plan économique. Au Canada, l'impact économique associé aux recettes touristiques du secteur culturel est appréciable. En 2007, le produit intérieur brut découlant du tourisme culturel, défini comme les activités liées aux arts, au patrimoine et aux Autochtones, s'élevait à 5,1 milliards de dollars et produisait un revenu de travail d'environ 3,3 milliards de dollars (The Outspan Group Inc. et al., 2009). D’après cette même étude, le tourisme culturel apportait $16 \%$ des recettes touristiques au pays (idem).
En 2006, l'Enquête sur les activités et les préférences en matière de voyage a été commanditée par 14 organismes fédéraux et provinciaux, dont le ministère du Tourisme du Québec ${ }^{2}$. Des voyageurs ayant effectué des séjours d'une nuit et plus au cours des deux années précédant l'enquête ont été interrogés, et l'échantillon était représentatif de la population du Canada et des États-Unis. L'enquête a permis d'obtenir de l'information sur environ 200 activités différentes pratiquées par les répondants lors de leurs voyages.

Aux fins d'analyse, les activités ont été regroupées dans plusieurs grandes catégories, dont celle des "visites de sites historiques, de musées et de galeries d'art». Les activités suivantes sont les plus importantes dans cette grande catégorie :

- promenades dans une ville pour examiner les bâtiments et l'architecture;

- visites de sites ou de bâtiments historiques de renom;

- visites d'autres sites, monuments et bâtiments historiques;

- visites de sites ou de lieux naturels de renom;

- visites de musées d'histoire ou de patrimoine;

- galeries d'art.

Parmi les voyageurs d'agrément canadiens, $58 \%$ ont effectué au moins une activité dans cette catégorie lors d'un voyage au cours des deux années précédentes. Ce pourcentage a été de $53 \%$ chez les voyageurs américains. Exception faite du magasinage et des sorties au restaurant, cette catégorie d'activités a été la plus fréquemment pratiquée par les voyageurs, soit davantage que les autres types d'activités culturelles et de divertissement tels les parcs thématiques et les expositions, les foires et festivals ou les casinos (Lang Research Inc., 2007a : 6; Lang Research Inc., 2007b : 4).

Parmi les personnes qui ont pratiqué une activité dans la catégorie «visites de sites historiques, des musées et des galeries d'art», $29 \%$ des voyageurs canadiens et $33 \%$ des voyageurs américains ont déclaré que la pratique d'une de ces activités a été la raison principale d'au moins un voyage effectué au cours des deux années précédentes. 


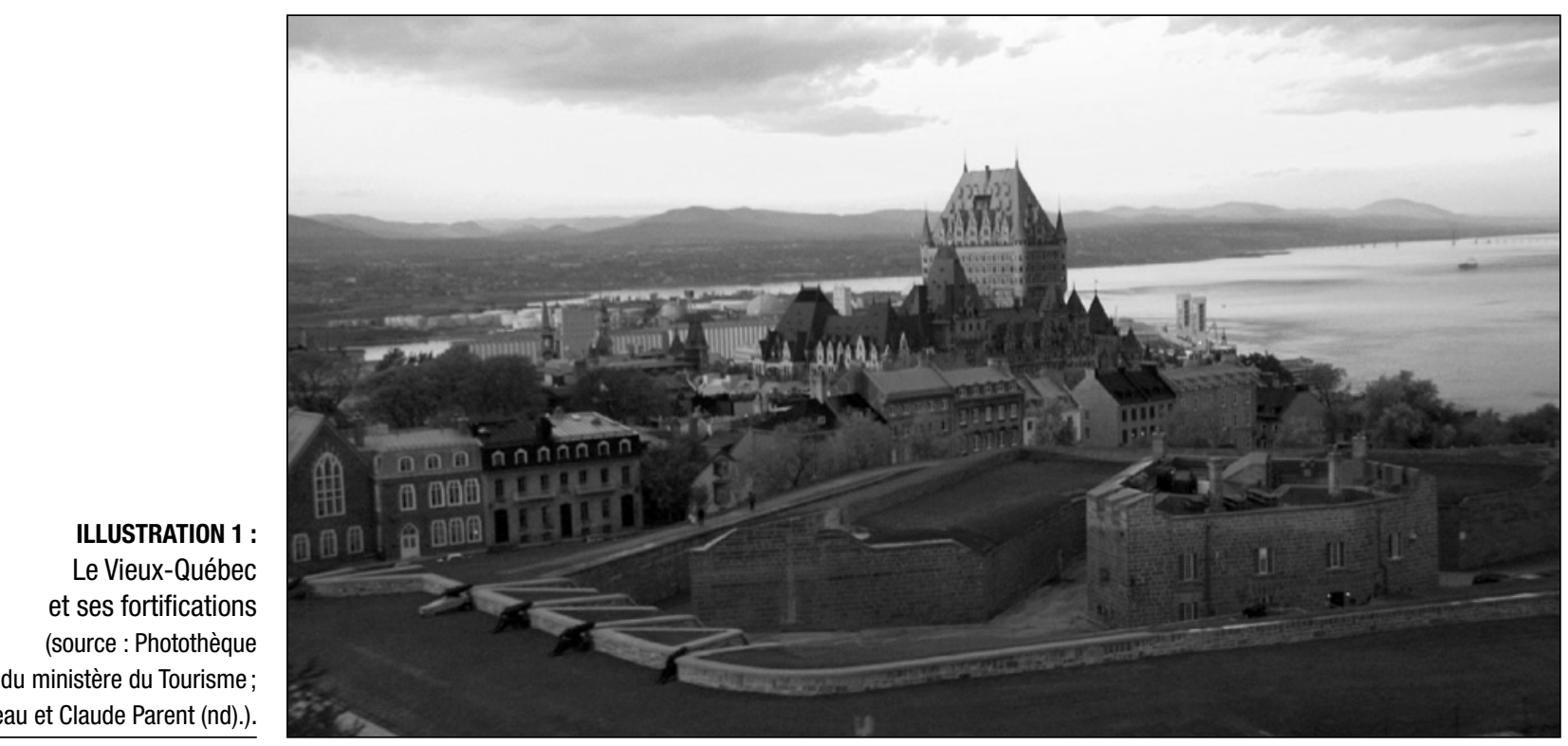

De façon générale, les voyageurs canadiens semblent favoriser davantage ce genre d'activités que les voyageurs américains. En revanche, parmi les personnes qui les pratiquent, c'est pour les voyageurs américains que ces activités constituent le plus souvent la raison principale d'effectuer un voyage.

Il est à noter aussi que les sites ou les bâtiments historiques de renom et les merveilles naturelles de renom sont cités plus souvent comme la raison principale d'un voyage par les gens qui visitent ces lieux, lorsqu'on les compare aux promenades urbaines, aux visites d'autres sites, monuments ou bâtiments historiques et aux visites de musées d'histoire ou de patrimoine. On peut en déduire que les sites ayant une certaine renommée exercent un attrait qui motive davantage le déplacement des voyageurs, ce qui renforce la pertinence de réfléchir sur les impacts de la reconnaissance d'un site comme patrimoine mondial. Ces études réalisées à des échelles et selon des points de vue variés donnent pour preuve que le tourisme culturel ne doit pas être négligé.

\section{Enjeux du développement durable}

L'importance des sites patrimoniaux dans la pratique touristique n'est plus à démontrer. Tel est le cas des sites du patrimoine mondial qui font partie des attractions touristiques les plus visitées (WTO, 2009). En raison de cette haute fréquentation, ces sites risquent de se détériorer si l'on n'y prend garde. Pour cette raison, la conservation du patrimoine, culturel ou naturel, passe par le développement durable (Zuindeau, 2010). Si un site patrimonial devient trop fréquenté par le tourisme, le risque est élevé qu'il se détériore, ce qui génèrerait alors une diminution de son attrait. Ceci concerne certains sites patrimoniaux qui ne sont pas forcément conçus dès leur origine pour recevoir un grand nombre de visiteurs et où l'on observe une forte concentration de touristes sur une relativement petite superficie (Pedersen, 2009). Lorsque l'accès (routes), les moyens de transport mis à disposition des visiteurs, les déchets générés par la fréquentation du tourisme de masse et l'hébergement réservé aux touristes ne sont pas gérés de manière adéquate, le risque de dégradation du site patrimonial est élevé. On se rend compte alors de la nécessité et de la valeur de la mise en place de stratégies prenant en compte les principes du développement durable. De plus, ces stratégies peuvent bénéficier à la population locale.

Notons à ce propos que le ministère du Tourisme intègre dans sa politique des principes de développement durable (Ministère du Tourisme, 2005). En 2006, le gouvernement du Québec a adopté la Loi sur le développement durable (L.R.Q., chapitre D-8.1.1). Cette dernière instaure le nouveau cadre de gestion axé sur le développement durable pour l'ensemble de l'administration publique. Cette loi énumère 16 principes qui servent de guide pour mieux intégrer la recherche de développement durable dans les interventions. Dans le champ de cette chronique, nous relevons le principe de protection du patrimoine.

Selon ce principe, il s'agit ici de protéger le patrimoine culturel qui est "constitué de biens, de lieux, de paysages, de traditions et de savoirs, [et] reflète l'identité d'une société. Il transmet les valeurs de celle-ci de génération en génération et sa conservation favorise le caractère durable du développement. Il importe d'assurer son identification, sa protection et sa mise en valeur, en tenant compte des composantes de rareté et de fragilité qui le caractérisent » (Ministère du Développement durable, de l'Environnement et des Parcs, 2009: 5).

\section{Développement durable et patrimoine : une double association}

Si la gestion de sites patrimoniaux doit inévitablement intégrer la prise en compte de principes du développement durable, on peut se demander si, à l'inverse, la mise en lumière de sites patrimoniaux, qu'ils soient reconnus par l'UNESCO ou non, peut contribuer au développement durable (Vereczi, 2009). 


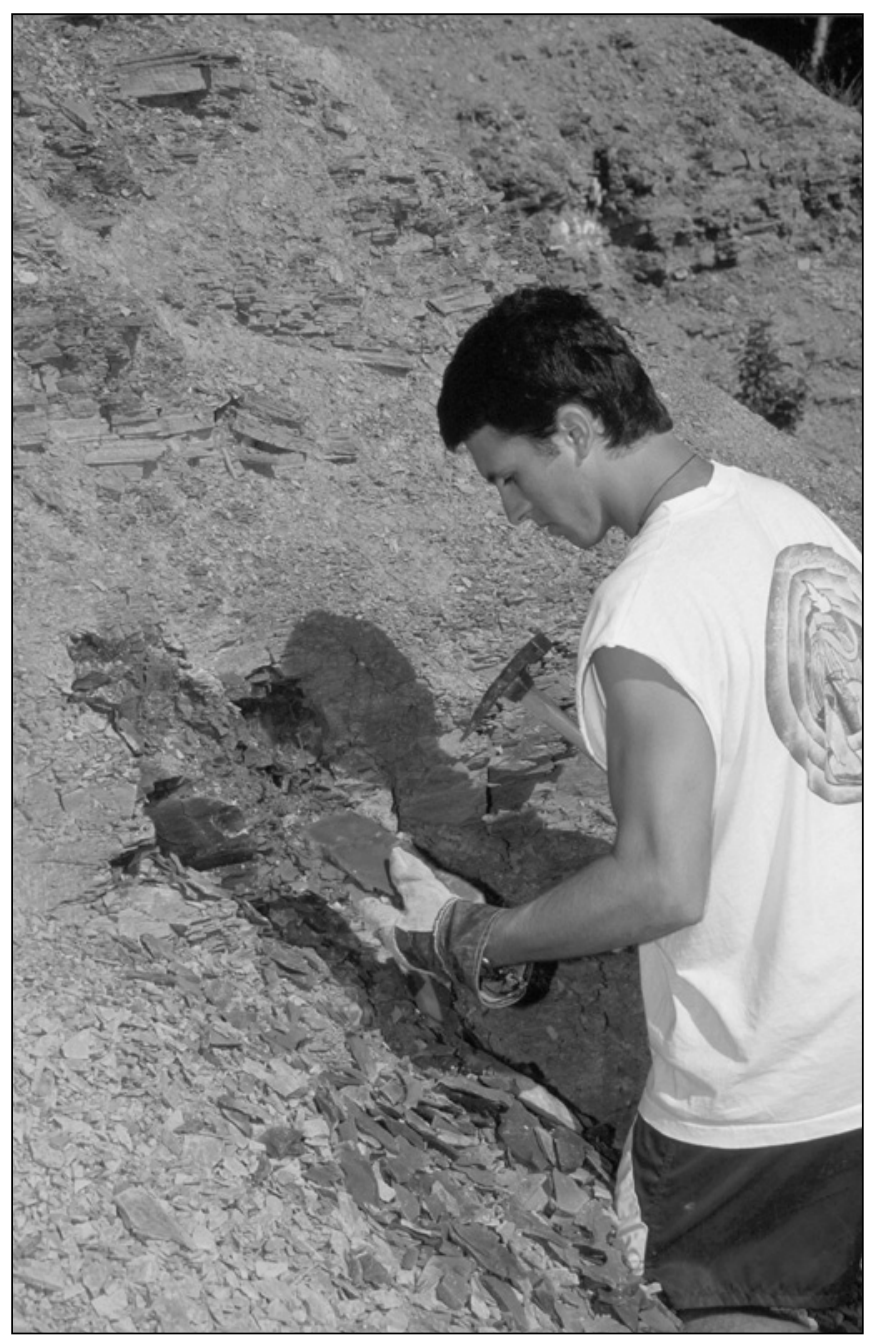

ILLUSTRATION 2 : Musée d'histoire naturelle du parc national de Miguasha (source : Photothèque du ministère du Tourisme, Jean-Pierre Huard (nd)).

En d'autres termes, la conservation du patrimoine pourrait générer le développement du tourisme culturel et le développement durable. Quelques exemples sont présentés dans les lignes qui suivent.

Certains artisans perpétuent des savoir-faire, c'est-à-dire du patrimoine immatériel, et œuvrent comme témoins de traditions locales. Ce faisant, ils soutiennent également l'économie locale, d'autant plus lorsque leurs matières premières proviennent des ressources de la région. Par ailleurs, certains de ces artisans proposent les articles qu'ils produisent aux visiteurs de passage, dans un commerce attenant à leur atelier ou des commerces répartis dans leur région. Tel est le cas des Économusées ${ }^{\circ}$ qui s'exposent à la vue du public en activité de fabrication et qui vendent les produits ainsi préparés. On peut découvrir ainsi des techniques aussi diverses que le fumage du poisson, le soufflage du verre ou le tannage du cuir. Certains de ces savoir-faire tomberaient en désuétude et dans l'oubli sans cette mise en valeur auprès des touristes, qui a pour effet de sensibiliser les résidents et de préserver un héritage patrimonial. Par ce type d'activités, les principes, découlant de la Loi sur le développement durable, de conservation du patrimoine culturel, d'efficacité économique, de production et de consommation responsables sont respectés.

Notons encore les nombreux festivals qui mettent en vedette les contes, la musique ou les danses traditionnelles, de même que ceux qui célèbrent la gastronomie locale. Ils participent ainsi à la diffusion du patrimoine immatériel et contribuent à la recherche d'authenticité et d'unicité du visiteur. On peut prendre pour exemple les pow-wow, les fêtes de la Nouvelle-France (à Québec) ou le festival du Bleuet (à Dolbeau-Mistassini). À cela s'ajoutent les activités liées à l'agrotourisme qui favorisent tant la production que la consommation de produits locaux.

En outre, les visites et les tours guidés à pied, que ce soit accompagné d'une brochure, d'un chemin tracé sur le sol, d'un audioguide, d'un MP3 ou de tout autre moyen technique ou bien sûr d'un guide en chair en os, permettent de faire une empreinte environnementale moindre en comparaison avec des tours guidés en autobus. De plus, cette manière d'approcher les sites patrimoniaux favorise la proximité tant aux sites eux-mêmes qu'aux commerces qui les environnent.

\section{Conclusion}

$\mathrm{Si}$, au Québec, les deux sites du patrimoine mondial, le VieuxQuébec (voir illustration 1) et le parc national de Miguasha (voir illustration 2), exigent l'intégration du développement durable dans les pratiques touristiques, ils présentent des particularités contrastées. D’une part, le Vieux-Québec est très connu et il attire des foules qu'il importe de gérer comme nous l'avons exposé précédemment. D'autre part, le site de Miguasha, même s'il contient des fossiles uniques au monde, est encore peu renommé et les visiteurs qui le fréquentent ne sont pas aussi nombreux (26000 visiteurs par an, Sépaq, 2011).

Malgré les bénéfices que peuvent apporter à une région la valorisation du patrimoine, cette dernière se doit d'éviter l'écueil de la disneylandisation (Brunel, 2006a) ou de la dérive du tout patrimonialiser (Neyret, 2004). En premier lieu, la mise en scène d'un site pour correspondre aux attentes des touristes risque de porter atteinte à son authenticité, et par là même à son unicité (Brunel, 2006b). En second lieu, nommer patrimoine chaque maison, savoir-faire ou œuvre d'art entraîne les mêmes risques. Ces dérapages pourraient mener à une perte de sens des lieux et de l'identité des populations, ce qui irait à l'encontre d'un développement durable. Un cadre et une réflexion telle que la Loi sur les biens culturels (Gouvernement du Québec, 1985) peut être utile pour pallier ce problème. En somme, la réflexion sur la relation entre le développement durable et le patrimoine et plus particulièrement sur la mise en valeur du patrimoine comme agent de développement social, environnemental et économique mériterait d'être poursuivie.

\section{Notes}

1 Ce texte est le premier d'une nouvelle série de chroniques qui sera alimentée par la Direction de l'évaluation et de l'analyse stratégique du ministère du Tourisme du Québec. 
2 Cette enquête a donné lieu à la publication de plus d'une centaine de rapports d'analyse en anglais qui sont regroupés sur le site Internet du ministère du Tourisme et de la Culture de l'Ontario : http:// www.mtc.gov.on.ca/en/research/travel_activities/tams. shtml. Plusieurs rapports ont été traduits en français et sont disponibles sur le site Internet du ministère du Tourisme du Québec : http://www.tourisme.gouv.qc.ca/publications/categorie/ rapports-enquete-activites-preferences-matiere-voyageseapv-2006-114.html.

\section{Références}

BRUNEL, Sylvie (2006a) La planète disneylandisée. Chroniques d'un tour du monde, Auxerre (France) : Sciences Humaines Éditions. 275 p.

BRUNEL, Sylvie (2006 b) «Quand le tourisme disneylandise la planète...", Sciences Humaines, vol. 174, p. 28-33.

GOUVERNEMENT DU QUÉBEC (1985) Loi sur les biens culturels, L.R.Q., chapitre B-4, Québec : Gouvernement du Québec.

GOUVERNEMENT DU QUÉBEC (2006) Loi sur le développement durable, L.R.Q., chapitre D-8.1.1, Québec: Gouvernement du Québec.

LANG RESEARCH INC. (2007a) Enquête sur les activités et les préférences en matière de voyages (EAPV) 2006, Marché touristique canadien, Profil des amateurs de visites de sites historiques, de musées et de galeries d'art durant des voyages d'une nuit ou plus. Rapport d'analyse. 27 p. <http://www.tourisme.gouv.qc.ca/publications/media/ document/etudes-statistiques/enquetes/Rapports-EAPV-Marchecanadien/Activites-culturelles-divertissement/FRAN-CDN-TAMS2006(Historical-Sites-Museums-Art-Galleries)09-03-17.pdf>, consulté le 8 septembre 2011.

LANG RESEARCH INC. (2007b) Enquête sur les activités et les préférences en matière de voyages (EAPV) 2006, Profils des activités des Américains, Visites de lieux historiques, des musées et des galeries d'art chez les touristes en voyage. $30 \mathrm{p}$. $<$ http://www.tourisme.gouv.qc.ca/publications/media/ document/etudes-statistiques/enquetes/Rapports-EAPV-Marcheamericain/Activites-culturelles-divertissement/US-Historical-SitesMuseums-Art-Galleries-fr.pdf $>$, consulté le 8 septembre 2011.

MINISTÈRE DE LA CULTURE, DES COMMUNICATIONS ET DE LA CONDITION FÉMININE (2011) Projet de loi $n^{\circ}$ 82. Loi sur le patrimoine culturel, Québec: Gouvernement du Québec.

MINISTÈRE DU DÉVELOPPEMENT DURABLE, DE L'ENVIRONNEMENT ET DES PARCS (2009) Guide pour la prise en compte des principes du développement durable, Québec: Gouvernement du Québec. 36 p.

MINISTĖRE DU TOURISME (2005) Vers un tourisme durable. Politique touristique du Québec, Québec : Gouvernement du Québec. 36 p.
NEYRET, Régis (2004) «Du monument isolé au "tout patrimoine"», Géocarrefour, vol. 79, n 3, p. 231-237.

ORGANISATION DE COOPÉRATION ET DE DÉVELOPPEMENT ÉCONOMIQUES (2009) The Impact of Culture on Tourism, Paris : OCDE. $156 \mathrm{p}$.

PEDERSEN, Art (2009) «The World Heritage Sustainable Tourism Programme: Issues and Strategies for Enhancing Coordination for Tourism Management at WHS», DANS Sustainable Tourism Management at World Heritage Sites. Enhancing Inter-agency and Stakeholder Coordination for Joint Action. International Conference, Huangshan, China, 24-27 March 2008, sous la direction de WORLD TOURISM ORGANIZATION, p. 96-112. Madrid : World Tourism Organization.

SÉPAQ (2011) Parc national de Miguasha, site du réseau de la Sépaq, $<$ http://www.sepaq.com/pq/mig/decouvrir/portrait.dot $>$, consulté le 8 septembre 2011.

THE OUTSPAN GROUP INC. ET RESEARCH RESOLUTIONS AND CONSULTING LTD. (2009) L'impact économique du tourisme culturel et sportif au Canada, Ottawa : Ministère du Patrimoine canadien et Industrie Canada. $40 \mathrm{p}$.

VERECZI, Gabor (2009) «Key Challenges and Options for Sustainable Tourism at World Heritage Sites». DANS Sustainable Tourism Management at World Heritage Sites. Enhancing Inter-agency and Stakeholder Coordination for Joint Action. International Conference, Huangshan, China, 24-27 March 2008, sous la direction de WORLD TOURISM ORGANIZATION, p. 75-95. Madrid : World Tourism Organization.

VILLE DE MONTRÉAL (2004) Énoncé d'orientation pour une politique du patrimoine. Rapport du groupe conseil, Montréal : Ville de Montréal. $40 \mathrm{p}$.

WORLD TOURISM ORGANIZATION (2009) «The Huangshan Declaration - Summary of Key Conclusions and Recommandations", DANS Sustainable Tourism Management at World Heritage Sites. Enhancing Inter-agency and Stakeholder Coordination for Joint Action. International Conference, Huangshan, China, 24-27 March 2008, sous la direction de WORLD TOURISM ORGANIZATION, p. 3-10. Madrid : World Tourism Organization.

ZUINDEAU, Bertrand (2010) Préface, DANS Ressources, patrimoine, territoires et développement durable, sous la direction de Muriel MAILLEFERT, Olivier PETIT et Sandrine ROUSSEAU, p. 9-13. Bruxelles : P.I.E. Peter Lang S.A. 